\title{
THERMODYNAMICS OF LARGE-SCALE HETEROGENEOUS WIRELESS NETWORKS
}

\section{David Tse}

03/06/2014

Final Report 


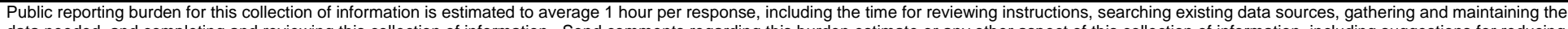

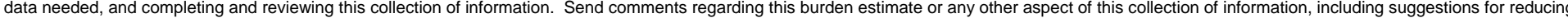

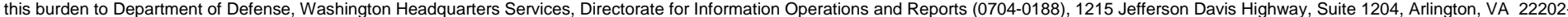

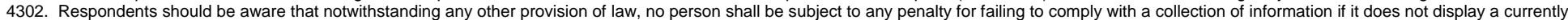
valid OMB control number. PLEASE DO NOT RETURN YOUR FORM TO THE ABOVE ADDRESS.

\begin{tabular}{l|l|l} 
1. REPORT DATE (DD-MM-YYYY) & 2. REPORT TYPE & 3. DATES COVERED (FrOm - To)
\end{tabular} 28-02-2014 final

4. TITLE AND SUBTITLE

Thermodynamics of Large-Scale Heterogeneous Wireless Networks From 01-05-2009 to 30-11-2013 5a. CONTRACT NUMBER FA9550-09-1-0317 5b. GRANT NUMBER FA9550-09-1-0317 5c. PROGRAM ELEMENT NUMBER

\section{AUTHOR(S)}

PI: David Tse, U.C. Berkeley co-PI: Piyush Gupta, Alcatel-Lucent Bell Labs co-PI: Devavrat Shah, MIT

5d. PROJECT NUMBER

5e. TASK NUMBER

5f. WORK UNIT NUMBER

\section{PERFORMING ORGANIZATION NAME(S) AND ADDRESS(ES)}

University of California, Berkeley CA

8. PERFORMING ORGANIZATION REPORT NUMBER

Alcatel-Lucent Bell Labs, Murray Hill, New Jersey

MIT, Cambridge, MA

9. SPONSORING I MONITORING AGENCY NAME(S) AND ADDRESS(ES)

10. SPONSOR/MONITOR'S ACRONYM(S)

DAF-AFOSR

ARO

11. SPONSOR/MONITOR'S REPORT NUMBER(S)

\section{DISTRIBUTION I AVAILABILITY STATEMENT}

\section{SUPPLEMENTARY NOTES}

\section{ABSTRACT}

In recent years, large wireless networks have become the architecture of choice in many emerging scenarios, such as mesh networks for providing infrastructure in metro areas, communication infrastructure under extreme conditions in military applications, cost-effective sensor networks for monitoring and surveillance, peer-to-peer networks between handheld devices in a social setup, among others. A fundamental issue is to determine the best performance that can be achieved in such networks. This is the theme of the project. In particular, we have investigated in depth: capacity, synchronization, in-network computation, distributed medium access and the role of feedback. Towards the latter part of this project, we have applied the lessons we learnt about wireless networks to other types of networks such as power networks.

\section{SUBJECT TERMS}

LIBS, IR fluorescence

\begin{tabular}{|c|c|c|c|c|c|}
\hline \multicolumn{3}{|c|}{ 16. SECURITY CLASSIFICATION OF: } & \multirow{2}{*}{$\begin{array}{l}\text { 17. LIMITATION } \\
\text { OF ABSTRACT } \\
\text { UU }\end{array}$} & \multirow{2}{*}{$\begin{array}{l}\text { 18. NUMBER } \\
\text { OF PAGES } \\
4\end{array}$} & \multirow{2}{*}{$\begin{array}{l}\text { 19a. NAME OF RESPONSIBLE PERSON } \\
\begin{array}{l}\text { 19b. TELEPHONE NUMBER (include area } \\
\text { code) }\end{array}\end{array}$} \\
\hline $\begin{array}{l}\text { a. REPORT } \\
\text { UU }\end{array}$ & $\begin{array}{l}\text { b. ABSTRACT } \\
\text { UU }\end{array}$ & $\begin{array}{l}\text { c. THIS PAGE } \\
\text { UU }\end{array}$ & & & \\
\hline
\end{tabular}




\title{
Thermodynamics of Large-Scale Heterogeneous Wireless Networks FA9550-09-1-0317 Final Report
}

\author{
PI: David Tse, co-PIs: Piyush Gupta, Devavrat Shah
}

In recent years, large wireless networks have become the architecture of choice in many emerging scenarios, such as mesh networks for providing infrastructure in metro areas, communication infrastructure under extreme conditions in military applications, cost-effective sensor networks for monitoring and surveillance, peer-to-peer networks between handheld devices in a social setup, among others. A fundamental issue is to determine the best performance that can be achieved in such networks. This is the theme of the project. In particular, we have investigated in depth: capacity, synchronization, in-network computation, distributed medium access and the role of feedback. Towards the latter part of this project, we have applied the lessons we learnt about wireless networks to other types of networks such as power networks. More details below.

\section{CAPACITY}

Finding the capacity region of a wireless network is an important but also well-known intractable problem. Research has thus focused on the scaling analysis, which determines the scaling of performance as the number of nodes in the network increases. This approach has led to significant progress in recent years, but this progress has come at the cost of making various homogeneity assumptions about the network, such as uniform node placement, uniform traffic demands, and uniform service requirements. In the first part of this project, we focused on relaxing these constraints, allowing for more heterogeneous network settings. In particular, we characterized how non-uniform node placement [2], [3], non-uniform traffic demands [1], varied service requirements [8], and energy constraints [4] impact performance scaling of wireless networks. Each of these heterogeneities is described by many micro parameters: For example, a non-uniform node placement is described by the position of each individual node in the network; a non-uniform traffic demand is described by the traffic for each individual node pair. As a key engineering insight, we derived for each of these heterogeneous network settings a significantly smaller number of macro parameters that provide a sufficient description of the heterogeneity in terms of the scaling behavior. Thus, at a coarse level, these fewer parameters are enough to characterize the performance of the network.

The focus so far was on the behavior of wireless network performance as the number of users increases. We next turned our attention to the behavior of wireless networks with fixed number of users but increasing transmit power. The picture that emerges in this regime is quite different. Indeed, recent work by Avestimehr et al. showed that a compress-and-forward approach is approximately optimal in this regime of high transmit power, in contrast to multi-hop or cooperative communication schemes that are approximately optimal in the regime of large number of users. However, neither of the approaches that is effective in one regime is also effective in the other one. In particular, the capacity gap of compress-and-forward grows quickly with increasing number of users in the network. Our goal in [7], [11] was to find a communication approach that is effective simultaneously in both the regimes, i.e., that simultaneously performs well in situations with high transmit powers and with large number of users. We proposed such an approach by combining recent work on compute-and-forward with ideas from interference alignment. The analysis revealed unexpected and deep connections to the field of Diophantine Approximations in Number Theory. 
Using these insights, we developed communication schemes that are provably approximately optimal for large networks and for high transmit powers.

\section{SYNCHRONIZATION}

A key issue to enable relaying/multi-hopping in wireless networks is synchronization between different network nodes. In traditional centralized scenarios, a central communication node, such as a base station in cellular wireless networks or an access point in wireless-lan, provides synchronization to all devices operating in its coverage area. In decentralized settings this is no longer possible, and the nodes have to establish synchronization among themselves. Establishing this synchronization incurs an energy overhead, and this overhead becomes significant in the energy-efficient low-power communication regime. Hence, there is a need for an energy-efficient synchronization framework. In [12], we developed energy efficient communication architectures requiring only limited synchronization. This is enabled through the design of novel modulation waveforms that can handle a carefully controlled amount of asynchronism between devices. We showed that the proposed scheme is asymptotically optimal for the Gaussian channel with synchronization errors in terms of energy efficiency as measured by the rate per unit energy. In the process, we also established that the lack of synchronization causes negligible loss in energy efficiency. Generalizing this framework, in [13], we developed energy-efficient strategies for communication over unsynchronized multiple-relay networks. We showed that in Gaussian diamond networks nearly full cooperative-communication gains (up to a constant factor) are feasible even with unsynchronized relay nodes.

\section{IN-NETWORK COMPUTATION}

In many networks, such as sensor networks or distributed computation networks, the key objective is not to communicate the entire data over the network but how to best perform in-network computation for data fusion. In [6], we studied the fundamental limits of communication requirement for in-network computation in wireless networks with localized information. For any function of independent sources, we derived a computable characterization of the rate region. We also showed that when computing symmetric functions of binary sources, certain additional information that is not needed in computing the function is inevitably learned, which led to new improved bounds for the minimum sum-rate that are better than the cut-set bounds. In such networks, it also is often desirable that the computed value of the function be kept secret from an eavesdropper. In [5], we introduced a Shannon-theoretic formulation to characterize a set of necessary and sufficient conditions for secure computability. Drawing on innate connections to the problem of secret-key generation using public communication, we showed that a function is securely computable if and only if its entropy is less than the "aided" secret-key capacity, for which we obtained a single-letter characterization.

To efficiently perform in-network computation in a distributed manner, in [9] we developed a queueingbased framework for computing a class of functions, FMux, which includes several useful functions such as parity, k-th order statistic, range functions, among others. For a general network performing these computations, we characterized the maximum refresh rate at which the network can operate in terms of the min-mincut of the network. In wireline networks, we showed that the maximum refresh rate is achievable by a simple algorithm that is dynamic, distributed, and only dependent on local information. In the case of wireless networks, we provided a MaxWeight-like algorithm with dynamic flow splitting, which is shown to be throughput-optimal.

\section{Distributed MEDiUM ACCESS}

Operationally, this means design of network-wide architecture that leads to efficient utilization of wireless resources. An important task in achieving this goal is the network-wide ability of different wireless transmission capable devices to coordinate transmissions with each other so as to utilize the resources most efficiently. In a large, unplanned network such as the existing WiFi networks in large public area 
(or even at home), such a problem is attempted to solve by means of the medium access protocol like the exponential back-off. However, such protocols are known to perform poorly in the context of large networks.

In [14]-[17], we have developed a new class of distributed medium access algorithms that resolve this long-standing challenge successfully. We describe summary of result next. Consider a wireless network of $n$ nodes represented by a graph $G=(V ; E)$ where an edge $(i, j) \in E$ models the fact that transmissions of $i$ and $j$ interfere with each other, i.e. simultaneous transmissions of $i$ and $j$ become unsuccessful. Hence it is required that at each time instance a set of non-interfering nodes (corresponding to an independent set in $G$ ) access the wireless medium. To utilize wireless resources efficiently, it is required to arbitrate the access of medium among interfering nodes properly. Moreover, to be of practical use, such a mechanism is required to be fully distributed as well as simple.

As the main result, we provide such a medium access algorithm. It is randomized, fully distributed and simple: each node attempts to access medium at each time with probability that is a function of its local information. We establish its efficiency by showing that the corresponding network Markov chain is positive recurrent as long as the demand imposed on the network can be supported by the wireless network (using any algorithm). In that sense, the proposed algorithm is optimal in terms of utilizing wireless resources. The algorithm is oblivious to the network graph structure, in contrast with classical solutions such as the so-called polynomial back-off algorithm that was established to be optimal only for a certain class of graphs. The key methodological innovations are (a) establishing the positive recurrence of coupled Markov chains, and (b) a comparison relation between stationary distributions of Markov chains building upon the classical Markov chain tree theorem.

Methodically, this work develops new class of distributed scheduling algorithm for any generic constrained queueing network. In general, there has recently been considerable interest in design of lowcomplexity, myopic, distributed and high-performance scheduling algorithms for constrained queueing network models that arise in the context of various emerging communication networks including the wireless network model of interest of this project, as well as data centers. For such large class of models, our algorithm is applicable. In summary, ours is a myopic, fully distributed and high-performance scheduling algorithm that performs few logical operations at each node per unit time.

\section{ROLE OF FEEDBACK}

Transmitter channel state information (CSIT) is crucial for the multiplexing gains offered by advanced interference management techniques in wireless networks, such as multiuser MIMO and interference alignment. Such CSIT is usually obtained by feedback from the receivers, but the feedback is subject to delays. The usual approach is to use the fed back information to predict the current channel state and then apply a scheme designed assuming perfect CSIT. When the feedback delay is large compared to the channel coherence time, such a prediction approach completely fails to achieve any multiplexing gain. In this regime, the conventional wisdom is that the fed back channel state information is completely useless. In [18], we show that even in this case, the completely stale CSI is still very useful. More concretely, we show that in a MIMO broadcast channel with $K$ transmit antennas and $K$ receivers each with 1 receive antenna, $\frac{K}{1+1 / 2+\ldots+1 / K}(>1)$ degrees of freedom is achievable even when the fed back channel state is completely independent of the current channel state. Moreover, we establish that if all receivers have independent and identically distributed channels, then this is the optimal number of degrees of freedom achievable. In the optimal scheme, the transmitter uses the fed back CSI to learn the side information that the receivers receive from previous transmissions rather than to predict the current channel state. Our result can be viewed as the first example of feedback providing a degree-of-freedom gain in memoryless channels.

\section{CAPACITY REGION OF POWER NETWORKS}

The optimal power flow (OPF) problem is perhaps the most basic optimization problem in power networks. The problem is to minimize the cost of power generation in a network to meet a given set 
of demands, while satisfying constraints on voltages and line power flows. While this problem has been studied for more than 30 years, most algorithms are heuristic and have no provable optimality property. In [19], we draw an analogy between power networks and wireless networks and ask: what is the capacity region of a power network? More specifically, we investigate the constraints on power flow in networks and its implications to the optimal power flow problem. The constraints are described by the injection region of a network; this is the set of all vectors of power injections, one at each bus, that can be achieved while satisfying the network and operation constraints. This is the power network analogy to the capacity region of power networks. If there are no operation constraints, we show the injection region of a network is the set of all injections satisfying the conservation of energy. If the network has a tree topology, e.g., a distribution network, we show that under voltage magnitude, line loss constraints, line flow constraints and certain bus real and reactive power constraints, the injection region and its convex hull have the same Pareto-front. The Pareto-front is of interest since these are the optimal solutions to the minimization of increasing functions over the injection region. For non-tree networks, we obtain a weaker result by characterizing the convex hull of the voltage constraint injection region for lossless cycles and certain combinations of cycles and trees.

\section{REFERENCES}

[1] U. Niesen, P. Gupta, D. Shah: The Balanced Unicast and Multicast Capacity Regions of Large Wireless Networks, IEEE Transactions on Information Theory, May 2010.

[2] U. Niesen, P. Gupta, D. Tse: On the Optimality of Multi-Hop Communication in Large Wireless Networks, IEEE International Symposium on Information Theory, June 2010.

[3] U. Niesen, P. Gupta, D. Tse: A Communication Architecture for Large Heterogeneous Wireless Networks, Allerton Conference, September 2010.

[4] S. Kamath, U. Niesen, P. Gupta: The Capacity Per Unit Energy of Large Wireless Networks, IEEE International Symposium on Information Theory, July 2011.

[5] H. Tyagi, P. Narayan, P. Gupta: When is a function securely computable?, IEEE Transactions on Information Theory, vol. 57, no. 10, pp. 6337-6350, October 2011.

[6] N. Ma, P. Ishwar, P. Gupta: Interactive source coding for function computation in collocated networks, IEEE Transactions on Information Theory, vol. 58, no. 7, pp. 4289-4305, July 2012.

[7] U. Niesen, P. Whiting: The Degrees of Freedom of Compute-and-Forward, IEEE Transactions on Information Theory, August 2012.

[8] U. Niesen, D. Shah, G. Wornell: Caching in Wireless Networks, IEEE Transactions on Information Theory, October 2012.

[9] S. Banerjee, P. Gupta, S. Shakkottai: Towards a queueing-based framework for in-network function computation, Queueing Systems, Vol 72, Issue 3-4, pp 219-250, December 2012.

[10] N. Karamchandani, U. Niesen, S. Diggavi: Computation over Mismatched Channels, IEEE Journal on Selected Areas in Communications, April 2013.

[11] U. Niesen, B. Nazer, P. Whiting: Computation Alignment: Capacity Approximation without Noise Accumulation, IEEE Transactions on Information Theory, June 2013.

[12] Y.-C. Huang, U. Niesen, P. Gupta: Energy-Efficient Communication in the Presence of Synchronization Errors, IEEE International Symposium on Information Theory, July 2013; also submitted to IEEE Transactions on Information Theory, January 2013.

[13] R. Kolte, U. Niesen, P. Gupta: Energy-Efficient Communication over the Unsynchronized Gaussian Diamond Network, submitted to IEEE Transactions on Information Theory, January 2014.

[14] S. Rajagopalan, D. Shah and J. Shin, Network adiabatic theorem: an efficient randomized protocol for contention resolution, in Proceedings of ACM Sigmetrics, 2009. (Best Student Paper Award).

[15] L. Jiang, D. Shah, J. Shin and J. Walrand, Distributed random access algorithm: scheduling and congestion control, IEEE Transactions on Information Theory, 2010.

[16] P. Tetali, D. Shah and J. Shin, Medium access using queues, in Proceedings of IEEE FOCS, 2011.

[17] D. Shah and J. Shin, Randomized scheduling algorithm for queueing networks, The Annals of Applied Probability, 2012. (Informs Applied Probability Society Best Publication Award for years 2010-2013.).

[18] M.A. Maddah-Ali and D. Tse, "Completely Stale Transmitter Channel State Information is Still Very Useful," IEEE Transactions on Information Theory, vol.58, no.7, pp.4418-4431, July 2012

[19] B. Zhang and D. Tse, "Geometry of Injection Region of Power Networks", Power Systems, IEEE Transactions on , vol.28, no.2, pp.788,797, May 2013. 